\title{
AMELIORATIVE EFFECT OF FRESH GARLIC AND VITAMIN E AGAINST LINEZOLID INDUCED HEPATO-RENAL OXIDATIVE DAMAGE IN RATS
}

\author{
HOSNY ABD EL FADIL ${ }^{1}$; SOHIER ABD EL LATIF ${ }^{1}$; AMANY BEHAIRY ${ }^{2}$ and \\ AMANY HASSAN ${ }^{1,3}$ \\ ${ }^{1}$ Department of Pharmacology, Faculty of Veterinary Medicine, Zagazig University, Egypt. \\ ${ }^{2}$ Department of Physiology, Faculty of Veterinary Medicine, Zagazig University, Egypt. \\ FAX: +20- 55 - 228-3683 \\ ${ }^{3}$ Al-Ahrar Zagazig Teaching Hospital, Ministry of Health, Egypt.
}

Received: 30 June 2019; Accepted: 22 July 2019

\begin{abstract}
The present study was conducted to compare between the protective effect of either garlic or vitamin E (Vit E) against hepatic and renal damage induced by $100 \mathrm{mg} / \mathrm{kg}$ linezolid (LNZ) administration. Thirty male albino rats were allocated into equal six groups; first three control groups; (control, tween 80 and olive oil); Group4; received 100mg LNZ / $\mathrm{kg}$ for 14 consecutive days. The last two groups were administered either garlic (500 $\mathrm{mg} / \mathrm{kg})$ or Vit E (100 mg/kg) prior to LNZ administration daily for 14 consecutive days. Blood samples were obtained from all rats for biochemical investigations and oxidative stress biomarkers. Specimens from liver and kidneys were excised for histopathological investigation. Linezolid induced a significant elevation in serum Aspartate aminotransferase (AST), Alanine aminotransferase (ALT), Alkaline phosphatase (ALP) and urea. Alterations in antioxidant / oxidant status were noticed during LNZ administration. Administration of garlic or Vit E modulated the previous parameters and restored histological structures. In conclusion, both garlic and Vit E were able to ameliorate hepato-renal alterations induced by LNZ via their antioxidant effects.
\end{abstract}

Key words: Linezolid, Garlic, Vitamin E, Kidney Liver.

\section{INTRODUCTION}

Recently, the broad activity of linezolid (LNZ) against gram- positive pathogens enables it to be a commonly prescribed antibiotic in clinics. Little studies were conducted to manage toxicities related to LNZ. Linezolid is an oxazolidinone antibiotic, it acts broadly against gram-positive micro-organisms via inhibition bacterial protein synthesis. It contorts the tRNA binding site overlapping the two ribosomal subunits $50 \mathrm{~S}$ and $30 \mathrm{~S}$ and inhibits protein synthesis before it starts (Tripati, 2014).

Linezolid oral bioavailability is a round $100 \%$, permitting a move from the intravenous administration to the oral route without dose adjustment (Hombal et al., 2017). Hepatotoxicity (Vivekanandan et al., 2018), renal dysfunction (Takahashi et al., 2011), optic neuropathy, lactic acidosis (Carbajo et al., 2011) and bone marrow suppression (Bishop et al., 2006) are LNZ- associated adverse effects. Vinh and Rubinstein, (2009)

Corresponding author: Dr. AMANY BEHAIRY E-mail address: amanybehairy25688@gmail.com Present address: Department of Physiology, Faculty of Veterinary Medicine, Zagazig University, Egypt. FAX: +20- 55-228-3683 demonstrated that LNZ use is associated with disturbances in liver enzymes. Linezolid treated rats showed a significant elevation in lipid peroxidation markers such as MDA in the liver and cause free radical-induced liver damage (Vivekanandan et al., 2018). LZD represses cell proliferation and delays the cellular metabolic activity by influencing the mitochondrial function (Duewelhenke et al., 2007).

Natural origin, weak side effects and cost effectiveness expand the use of natural supplements and medicinal plants in treatment or even using them to alleviate the adverse action of certain drugs. There are little studies which used natural antioxidants to alleviate LNZ detrimental effects. Garlic (Allium sativum), a member of the Liliaceae family, is one of the most popular herbs used in modern folkloric medicine (Chinnala et al., 2018). Garlic is commonly used as immunomodulatory, hepatoprotective, (Uma et al., 2007), and antioxidant agent (Schäfer and Kaschula, 2014). Garlic has two main classes of antioxidant machineries, flavonoids and sulfurcontaining compounds (diallyl sulfide, trisulfide and allyl-cysteine (Shirzad et al., 2011). Vitamin E (Vit E), a strong lipid-soluble antioxidant, counteracts reactive oxygen species (ROS) damage in polyunsaturated fatty acids and acts against damage caused to phospholipids as a membrane-stabilizing 
agent (Bradford et al., 2003).Vitamin E can prevent liver damage, fibrosis and cirrhosis progression during exposure to toxic substances (Sokol, 1996). The present work was conducted to study the ameliorative effect of garlic and Vit E on biochemical and histopathological disturbances associated with LNZ treatment in male albino rats in a trial to improve LNZ clinical use.

\section{MATERIALS AND METHODS}

\section{Reagents and drugs}

Linezolid (LNZ) tablets $(600 \mathrm{mg})$ obtained from Global Napi Pharmaceuticals Company, Egypt. Vitamin E (Vit E) capsules (1000 mg) purchased from Pharco Pharmaceuticals Company, Egypt.

Fresh garlic was obtained from local market and cleaned, washed. The cloves were peeled, weighed, added to suitable cold distilled water, grounded by mixer. The garlic homogenate was administered within 30 minutes of preparation.

\section{Animals management and designing}

Thirty apparently healthy adult male albino rats, weighing $200 \pm 10 \mathrm{~g}$ were obtained from the Laboratory Animal House, Faculty of Veterinary Medicine, Zagazig University, Egypt. The animals were housed in metal cages under optimal conditions. They were subjected to feed rodent diet ad libitum with free access to fresh water throughout the experimental period. Rats were acclimatized for 14 days before the experiment. The care and use of the animals confirmed to the rules of the institutional Animal Care and Use Committee of the Faculty of Veterinary Medicine, Zagazig University, Egypt.

After weighing the rats, they were randomly allocated into equal six groups $(n=5$ each). The first three groups were kept as control groups, $1^{\text {st }}$ group, rats was kept without any treatment. $2^{\text {nd }}$ group (tween 80 ), rats were given the diluting vehicle of LNZ (tween 80 ) in form of $0.5 \mathrm{ml} / 200 \mathrm{~g} /$ day. $3^{\text {rd }}$ group (olive oil), rats received the diluting vehicle of Vit $\mathrm{E}$ as 0.2 $\mathrm{ml}$ olive oil once daily. $4^{\text {th }}$ group (LNZ), rats administered 100mg LNZ/ kg/day (Aytan et al., 2009) dissolved in tween 80. $5^{\text {th }}$ group (garlic+ LNZ), rats were given $500 \mathrm{mg}$ fresh garlic $/ \mathrm{kg} /$ day (Banerjee et al., 2002) $1 \mathrm{~h}$ before LNZ dosing. $6^{\text {th }}$ group (Vit E+ LNZ), rats received $100 \mathrm{mg}$ Vit E $/ \mathrm{kg}$ /day (El-Demerdash et al., 2004) dissolved in olive oil $1 \mathrm{~h}$ before LNZ administration. All rats were administered their doses orally using oral gavage needle daily for 14 consecutive days.

\section{Blood sampling}

At $15^{\text {th }}$ day after the end of experiment, all rats were euthanized. Blood sample was collected in plain tube without anticoagulant, and then centrifuged at 3000 rpm for 10 minutes for serum collection until screening of liver and kidney damage biomarkers.

\section{Biochemical and oxidative status assessment}

Serum levels of aspartate aminotransferase (AST), alanine aminotransferase (ALT) were measured according to (Reitman and Frankel, 1957) and alkaline phosphatase (ALP) was estimated according to (Kind and King, 1954). Urea and creatinine were determined as previously described by (Fawcett and Scott, 1960) and (Henry et al., 1974) respectively. All parameters were measured using commercial kits (Diamond Diagnostic Company, Spinreact). Serum catalase (CAT), superoxide dismutase (SOD) and malondialdehyde (MDA) levels were estimated using commercial ELISA kits (Cusabio Biotech. Co., Ltd.,) by the methods of (Aebi, 1984), (Nishikimi et al., 1972) and (Ohkawa et al., 1979) respectively.

\section{Histopathological investigation}

After euthanization, specimens of liver and kidneys were collected from 5 rats /each group, fixed in $10 \%$ neutral buffered formalin solution. After that, they were embedded in paraffin, then $5 \mu \mathrm{m}$ sections were cut, stained with hematoxylin and eosin (H\&E) and finally examined under microscope.

\section{Statistical analysis}

The obtained data was represented as mean \pm SE. The values were analyzed by ANOVA followed by Duncan's test. The data were considered significant at $\mathrm{P} \leq 0.05$ (Tamhana and Dunlop, 2000).

\section{RESULTS}

Effect of garlic or Vit $E$ on biochemical and oxidative stress biomarkers in male albino rats treated with $\mathbf{L N Z}$

Data in Table 1 represented a significant increase in serum AST, ALT, ALP in rats orally administered LNZ comparing with control groups. The rats given garlic or Vit E with LNZ had significantly reduced AST, ALT, ALP levels as compared with LNZ treated rats. Urea levels showed a significant increase in LNZ treated rats in comparison with control groups. Garlic and Vit E ameliorated the increase in urea levels compared to LNZ group (Table 2). Creatinine concentration revealed a non significant change in all treated groups.

Compared to control groups, lipid peroxidation was markedly increased in LNZ treated group as represented by a significant increase in MDA level. Treatment with either garlic or Vit E expressed a significant decrease in MDA level comparing to LNZ group. Moreover, CAT and SOD were significantly decreased in LNZ treated rats comparing to control rats. Both CAT and SOD were significantly elevated in groups administered either garlic or Vit E (Fig 1). 
Table 1: Effect of garlic or Vit E on serum AST, ALT, ALP in male albino rats treated with LNZ.

\begin{tabular}{lccc}
\hline Groups & Parameters & & \\
\hline Control & AST $($ U/L) & ALT $($ U/L) & ALP ( U/L) \\
\hline Tween 80 & $19.66^{\mathbf{d}} \pm 0.88$ & $13.68^{\mathbf{d}} \pm 1.20$ & $72.10^{\mathbf{d}} \pm 1.37$ \\
\hline Olive oil & $17.5^{\mathbf{d}} \pm 3.5$ & $14.00^{\mathbf{d}} \pm 3.00$ & $71.30^{\mathbf{d}} \pm 0.40$ \\
\hline LNZ & $19.00^{\mathbf{d}} \pm 1.73$ & $13.00^{\mathbf{d}} \pm 1.53$ & $71.87^{\mathbf{d}} \pm 1.47$ \\
\hline Garlic+LNZ & $50.25^{\mathbf{a}} \pm 1.49$ & $54.75^{\mathbf{a}} \pm 2.01$ & $130.62^{\mathbf{a}} \pm 3.87$ \\
\hline Vit E+LNZ $^{\mathbf{b}} \pm$ & $38.00^{\mathbf{b}} \pm 1.82$ & $106.32^{\mathbf{b}} \pm 2.17$ \\
\hline Vas & $40.75^{\mathbf{b}} \pm 0.85$ & $23.75^{\mathbf{c}} \pm 1.49$ & $90.22^{\mathbf{c}} \pm 1.34$
\end{tabular}

Values are expressed as mean \pm SE. The different superscripts (a-d) for the same parameter are significantly different at $\mathrm{P} \leq 0.05$. AST: Aspartate aminotransferase; ALT: Alanine aminotransferase; ALP: Alkaline phosphatase; LNZ: Linezolid; Vit E: Vitamin E. $(\mathrm{n}=5)$.

Table 2: Effect of garlic or Vit E on serum urea and creatinine in male albino rats treated with LNZ.

\begin{tabular}{lcc}
\hline Groups & & \\
\hline Control & Urea $(\mathbf{m g} / \mathbf{d L})$ & Creatinine $(\mathbf{m g} / \mathbf{d L})$ \\
\hline Tween 80 & $27.46^{\mathbf{d}} \pm 0.99$ & $0.79^{\mathbf{a}} \pm 0.03$ \\
\hline Olive oil & $27.30^{\mathbf{d}} \pm 1.40$ & $0.76^{\mathbf{a}} \pm 0.02$ \\
\hline LNZ & $27.33^{\mathbf{d}} \pm 0.49$ & $0.79^{\mathbf{a}} \pm 0.02$ \\
\hline Garlic+LNZ & $60.67^{\mathbf{a}} \pm 1.95$ & $0.77^{\mathbf{a}} \pm 0.02$ \\
\hline Vit E+LNZ & $44.10^{\mathbf{b}} \pm 1.89$ & $0.77^{\mathbf{a}} \pm 0.02$ \\
\hline Val & $36.20^{\mathbf{c}} \pm 1.47$ & $0.74^{\mathbf{a}} \pm 0.04$ \\
\hline
\end{tabular}

Values are expressed as mean \pm SE. The different superscripts (a-d) for the same parameter are significantly different at $\mathrm{P} \leq 0.05$. LNZ: Linezolid; Vit E: Vitamin E. $(\mathrm{n}=5)$.

Figure 1

A

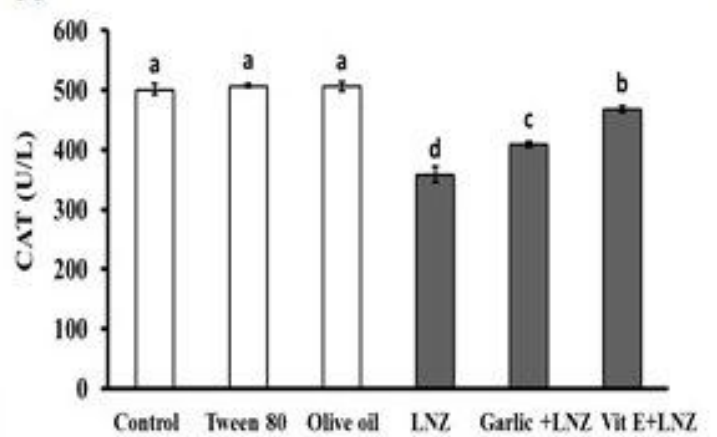

C

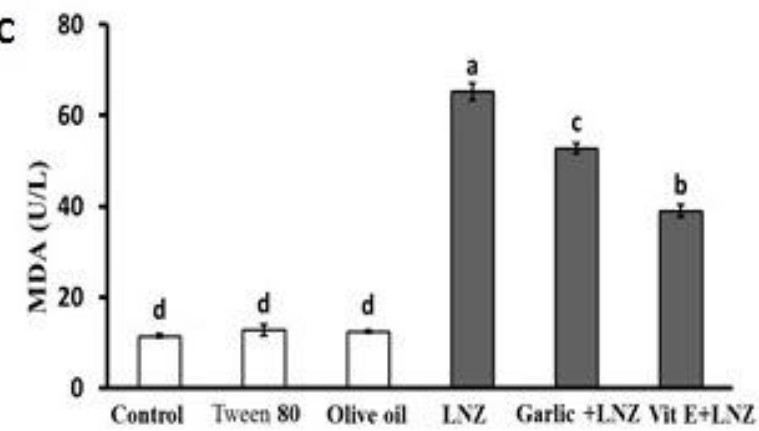

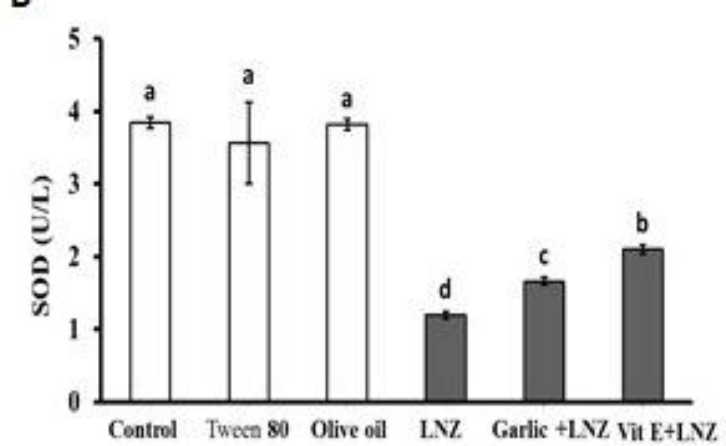

INZ Garbic +1NZ Nit E+LY

Figure 1: Effect of garlic and/or Vit $\mathrm{E}$ on antioxidant/oxidant status in male albino rats treated with LNZ. A: CAT (U/L). B: SOD (U/L). C: MDA (U/L). Values are expressed as mean \pm SE. Different letters above the bars indicant significant difference at $\mathrm{P} \leq$ 0.05. $(\mathrm{n}=5)$. CAT: Catalase; SOD: Superoxide dismutase; MDA: Malondialdehyde; LNZ: Linezolid; Vit E: Vitamin E. 


\section{Figure 2}
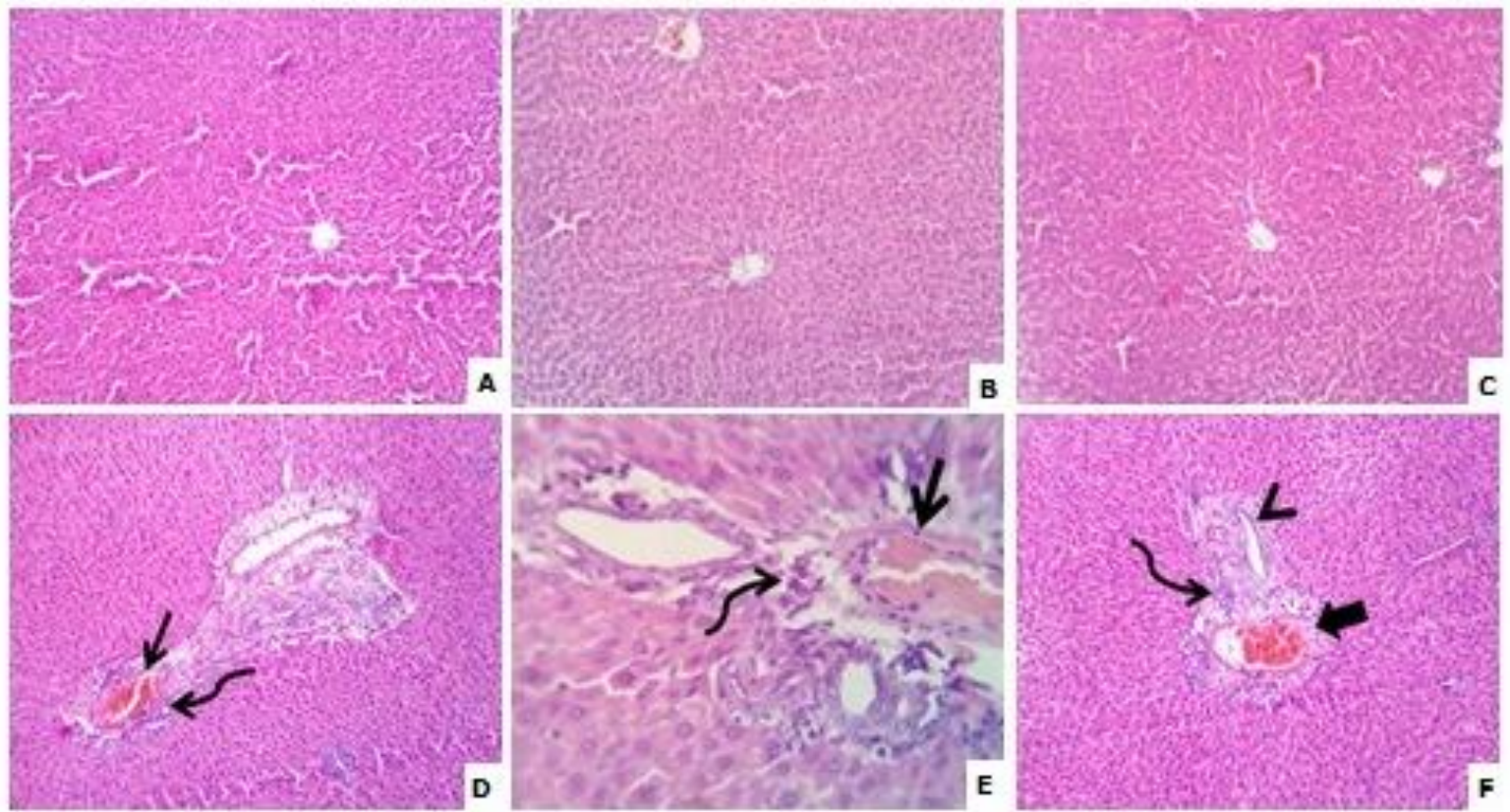

Figure 2: (A-F) Photomicrograph of hepatic H\&E stained sections, normal histological structures in control, tween 80 and olive oil group (A), (B) and (C) (H\&Ex100) respectively. Moderate congestion of hepatic portal blood vessels (open arrow) with moderate round cells infiltration (curved arrow) in LNZ group (D) (H\&Ex100). Garlic +LNZ group showed mild portal biliary proliferation, congestion (open arrow) and a few round cells (curved arrow) (E) (H\&Ex400). Mild congestion of hepatic blood vessels (thick arrow), round cells infiltration (curved arrow) and biliary proliferation (arrow head) in Vit E+ LNZ administered rats (F) (H\&Ex100).

\section{Figure 3}
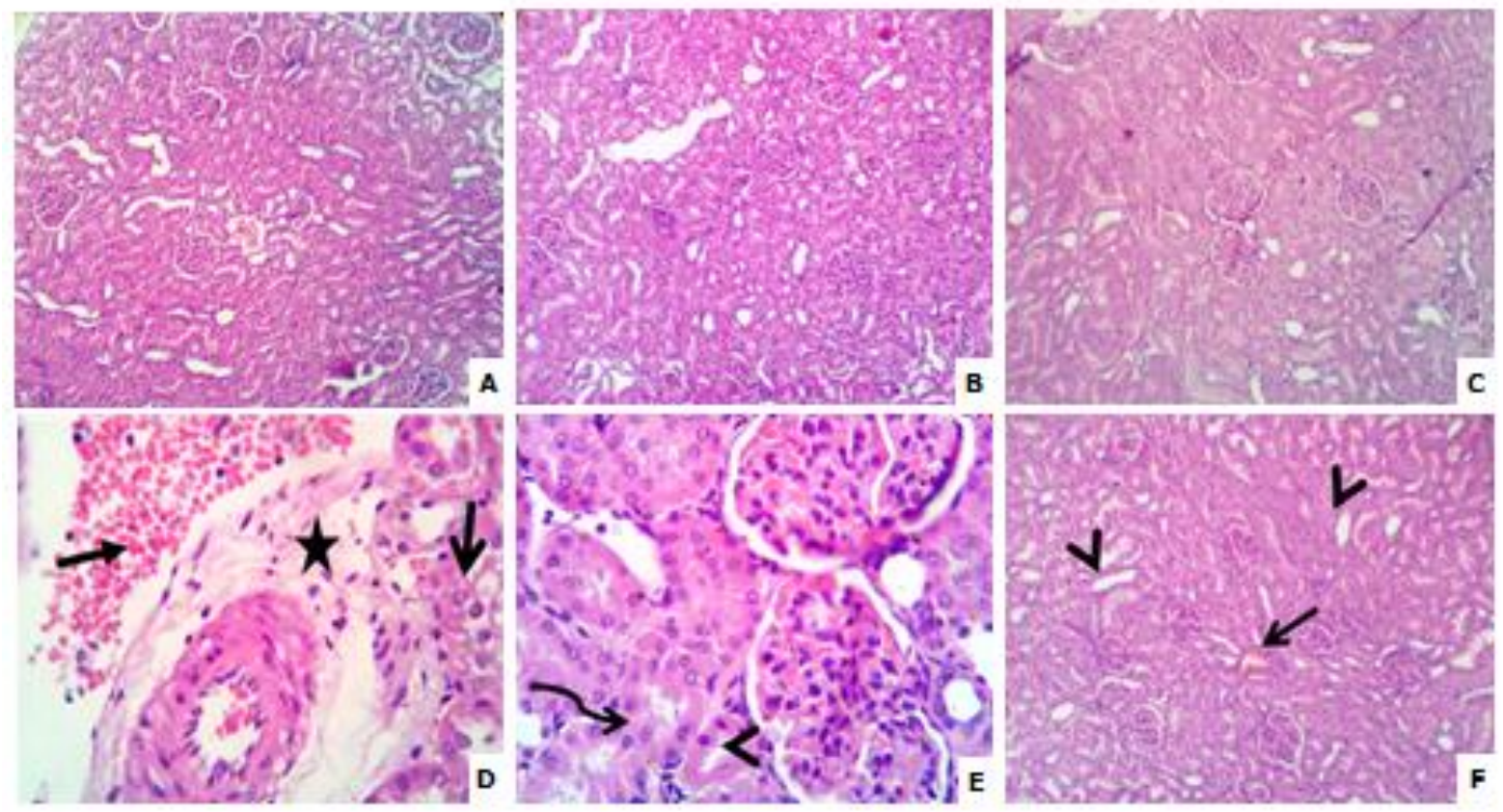

Figure 3: (A-F) Photomicrograph of renal tissue H\&E stained sections, normal histological findings in control, tween 80 and olive oil treated rats (A), (B) and (C) (H\&Ex100) respectively. LNZ administered rats showed perivascular edema (star), sometimes with hemorrhages (closed arrow). The renal tubular epithelium especially in the cortex showing degenerative changes (open arrow) (D) (H\&Ex400). Mild dilatation of some proximal and distal convoluted tubules and degenerative (curved arrow) changes (arrow head) in garlic+ LNZ group (E) (H\&Ex400). Mild congestion of some renal blood vessels (open arrow) and mild dilatation of some proximal and distal convoluted tubules (arrow heads) were observed in Vit E+LNZ treated rats (F) (H\&Ex100). 


\section{Histopathological findings}

\section{Liver}

Normal hepatic parenchyma with preserved lobular pattern, portal triads structures, vascular tree, kupffer cells and stromal component were observed in control groups (control, tween 80 and olive oil) (Fig. 2 A, b and c). Congestion of hepatic portal blood vessels and moderate round cells infiltration and biliary proliferation were seen in LNZ treated rats (Fig. 2D). Garlic+ LNZ treated group showed mild changes in portal area as mild portal biliary proliferation, congested portal blood vessels and few round cells infiltration (Fig. 2E). Meanwhile, normal structures with minimum residual tissue changes as mild congestion, round cells infiltration and biliary proliferation in the portal area of rats treated with Vit E prior to LNZ (Fig. 2F).

\section{Kidneys}

Light microscopical examination exhibited normal nephron units with preserved glomerular and tubular structures. The blood vessels and the stroma were within normal limits with normal histomorphology in the first control groups (control, tween 80 and olive oil) (Fig. 3 A, B and C) respectively. Linezolid treated rats showed modertae congestion of renal blood vessels with vacuolation of the vascular wall and perivascular edema, sometimes with hemorrhages, dilated collecting tubules. The renal tubular epithelium especially in the cortex showed degenerative changes mostly hydropic degeneration and necrotic changes (Fig. 3D). Mild dilatation of some proximal and distal convoluted tubules and mild degenerative changes in some renal tubular epithelium mostly cloudy swelling were seen in garlic+ LNZ treated group (Fig. 3E). Vit E+ LNZ group revealed preserved histomorphology of nephron units and preserved stroma with mild congestion of some renal blood vessels and mild dilatation of some proximal and distal convoluted tubules (Fig. 3F).

\section{DISCUSSION}

Linezolid is a preferable choice for many patients, not only for its broad activity against gram positive pathogens but also it has $100 \%$ bioavailability post its oral application. Linezolid associated deleterious actions including hepatic and renal oxidative disturbances restrict its prolong therapy. In this study we hypothesized that pretreatment with both garlic and Vit E for 14 successive days will adjust LNZ adverse actions and improve its clinical use.

Linezolid toxicity was considered to be the cause of the lactic acidosis (De Bus et al., 2010) which cause damage of the organs as liver and kidneys (Vivekanandan et al., 2018). The damage of hepatocytes causes leakage of the enzymes in the affected tissue and their release into the blood stream (Shaarawy et al., 2009). The serum levels of AST, ALT (indicators of liver function) and serum ALP (indicator of hepatobiliary disease) were unregulated in LNZ treated group indicating liver damage and this confirmed histopathologically. In agreement with animal studies of French, (2003) who demonstrated increases in hepatic transaminase with LNZ treatment which was dose-dependent and reversible.

Treatment with either garlic or Vit E protected the structural integrity of liver and improved most of histopathological changes represented by significant decrease in AST, ALT and ALP. Garlic extract has been shown to decrease liver enzymes in serum and prevent liver damage of rats with liver fibrosis (Nakagawat et al., 1989), due to its ability to reduce free radical-induced oxidative damage in the liver (Gedik et al., 2005). Vitamin E showed a significant decrease in the activity of these enzymes compared to cyclophosphamide treated group (Khanalizadeh and Najafian, 2014).

Urea and creatinine are waste products of protein metabolism, excreted by the kidney and their increase indicate renal function damage (Salem and Salem, 2016). During LNZ treatment, increased rate of LNZ non renal clearance because of decreased renal creatinine clearance. This compensatory increase for LNZ clearance due to enhanced drug biotransformation, biliary excretion, decreased absolute bioavailability, or changes in drug distribution (Brier et al., 2003). The existing study revealed a significant increase in urea which is sensitive indicator of renal abnormalities and that confirmed by histopathological examining, while non significant change in creatinine was observed in LNZ group compared to control groups. The serum creatinine is more sensitive kidney function test than urea (Chauhan et al., 2016). Because of increased creatinine tubular secretion, its serum level does not significantly increased until the glomerular filtration rate is reduced to less than $50 \%$ of normal level (Perrone et al., 1992). Urea is not secreted by the tubules, freely filtered by the glomerulus and 40-70\% is passively reabsorbed from the renal tubules (Newman and Price, 2005). Moreover, urea level is influenced by health status, feeding and liver function. The data are in accordance with Nukui et al. (2013) who reported renal function disturbance at $3^{\text {rd }}$ day of LNZ therapy. Either garlic or Vit E treatment modulated the urea alterations and confirmed by histological results. Garlic contains more than 200 chemical compounds including volatile oil with sulphur containing allicin, allin, ajone, allinase, peroxidase and myrosinase (Block, 1985). Vitamin E up-regulates of phospholipid A2 and cyclooxygenase1 , leads to the release of prostacyclin which improves the GFR and corrects the serum levels of creatinine and urea (Mohammed et al., 2014). 
The free radicals play a main role in numerous pathological dysfunctions such as hepatotoxicity, inflammations, diabetes mellitus and cardiovascular disorders (Zwart et al., 1999). SOD is the first enzyme in the antioxidant defense mechanism and CAT protects the cell from oxidative damage induced by $\mathrm{H}_{2} \mathrm{O}_{2}$ and hydroxyl radical. A significant reduction in CAT and SOD with a significant increase in MDA concentration were reported in LNZ treated rats compared with control groups and garlic or Vit $\mathrm{E}$ treatment ameliorates these alterations. The LNZ oxidative stress was previously confirmed by Vivekanandan et al. (2018) who reported a significant increase in lipid hydroperoxide and MDA with a significant decrease in SOD, CAT, glutathione reductase $(\mathrm{GSSH})$, peroxidase $(\mathrm{Px})$, and glutathione peroxidase $(\mathrm{Gpx})$. The present findings are in accordance with Zaidi et al. (2019) who reported that crude garlic extract significantly increased the hepatic activities of SOD, CAT and glutathione-S transferase (GST). The same results were obtained with aged garlic extract pretreatment in cisplatin treated rats (Nasr, 2014).

Such improvements for garlic are attributed to organosulfur compounds allicin, alliin, S-allyl cysteine (SAC) and S-allylmercaptocysteine (SAMC) which are potent free radical scavengers (Tsai et al., 2012) and (Mellon et al., 2000). Moreover, garlic increased resistance of low density lipoprotein to oxidation which accounts for its antioxidant and antiatherosclerotic functions (Lau, 2001). Vitamin E keeps the integrity of membrane by preventing lipid peroxidation and enhancing the activity of antioxidant enzymes (Abd et al., 2016). The antioxidant effect of Vit $\mathrm{E}$ is depend on the presence of a hydroxyl group in its phenolic group on the chromanol ring which can donate a hydrogen atom and, subsequently, neutralize the free radicals (Wang and Quinn, 1999).

\section{CONCLUSIONS}

This study was the first report to evaluate the protective effect of garlic or Vit E against LNZ induced hepato- renal oxidative stress. Linezolid induced biochemical disturbances and altered oxidative status and both garlic and Vit E restored liver and kidney function biomarkers and reestablished the antioxidant/oxidant condition. We recommend using either $\mathrm{Vit} \mathrm{E}$ or garlic as a supplement to alleviate LNZ undesirable actions.

\section{REFERENCES}

Abd, A.H.; Qasim, B.J.; Sahib, H.B. and Raheem, H. (2016): Nephroprotective Effect of Vitamin E and Origanum vulgare Extracts against Vancomycin Induced Nephrotoxicity in Rats. International Journal of Pharmaceutical Sciences Review and Research, 36(1): 89-96.
Aebi, H. (1984): catalase in vitro. Methods Enzymo 1.6: 105: 121-126.

Aytan, H.; Caliskan, A. C.; Yener, T.; Demirturk, F.; Aytan, P. and Yenisehirli, A. (2009): A novel antibiotic, linezolid, reduces intraperitoneal adhesion formation in the rat uterine horn model. Acta obstetricia et gynecologica Scandinavica, 88(7): 781-786.

Banerjee, S.K.; Dinda, A.K.; Manchanda, S.C. and Maulik, S.K. (2002): Chronic garlic administration protects rat heart against oxidative stress induced by ischemic reperfusion injury. BMC pharmacology, 2(1): 16.

Bishop, E.; Melvani, S.; Howden, B.P.; Charles, P.G.P. and Grayson, M.L. (2006): Good clinical outcomes but high rates of adverse reactions during linezolid therapy for serious infections: a proposed protocol for monitoring therapy in complex patients Antimicrob. Agents Chemother, 50: 1599-1602.

Block, E. (1985): The chemistry of garlic and onions. Sic Am., 252: 114-119.

Bradford, A.; Atkinson, J.; Fuller, N. and Rand, RP. (2003): The effect of vitamin $E$ on the structure of membrane lipid assemblies. The Journal of Lipid Research, 44(10): 1940-1945.

Brier, M.E.; Stalker, D.J.; Aronoff, G.R.; Batts, D.H.; Ryan, K.K.; O'Grady, M.; Hopkins, N.K. and Jungbluth, G.L. (2003): Pharmacokinetics of linezolid in subjects with renal dysfunction. Antimicrobial agents and chemotherapy, 47: 2775-2780.

Carbajo, T.; Fenollosa, M.; Pons, R. and Calvo, C. (2011): Lactic acidosis and linezolidinduced pancytopaenia Nefrologia, 31(1): 107-108.

Chauhan, P.; Yadav, R.; Kaushal, V. and Beniwal, P. (2016): Evaluation of serum biochemical profile of breast cancer patients. International Journal of Medical Research Health Sciences, 5,7: 1-7.

Chinnala, K.M.; Jayagar, P.P.; Motta, G.; Adusumilli, R.C. and Elsani, M.M. (2018): Evaluation of hepatoprotective activity of Allium sativum ethanolic extract in thioacetamide-induced hepatotoxicity in albino Wistar rats. American journal of research in medical sciences, 3(2): 48-53.

De Bus, L.; Depuydt, P.; Libbrecht, L.; Vandekerckhove, L.; Nollet, J.; Benoit, D.; Vogelaers, D. and Van Vlierberghe, $H$. (2010): Severe drug-induced liver injury associated with prolonged use of linezolid. Journal of Medical Toxicology, 6(3): 322-326.

Duewelhenke, N.; Krut, O. and Eysel, P. (2007): Influence on mitochondria and cytotoxicity of different antibiotics administered in high concentrations on primary human osteoblasts and cell lines. Antimicrobial Agents and Chemotherapy, 51(1): 54-63. 
El-Demerdash, F.M.; Yousef, M.I.; Kedwany, F.S. and Baghdadi, H.H. (2004): Role of $\alpha$ tocopherol and $\beta$-carotene in ameliorating the fenvalerate-induced changes in oxidative stress, hemato-biochemical parameters, and semen quality of male rats. Journal of Environmental Science and Health, Part B, 39(3): 443-459.

Fawcett, J.K. and Scott, J.E. (1960): A rapid and precise method for the determination of urea. $\mathrm{J}$ Clin Pathol., 13: 156-159.

French, G. (2003): Safety and tolerability of linezolid. Journal of Antimicrobial Chemotherapy, 51: 45-53.

Gedik, N.; Kabasakal, L.; Sehirli, O.; Ercan, F.; Sirvancl, S.; Keyer-Uysal, M. and Şener, G. (2005): Long-term administration of aqueous garlic extract (AGE) alleviates liver fibrosis and oxidative damage induced by biliary obstruction in rats. Life sciences, 76: 25932606.

Henry, R.J.; Canon, D.C. and Winkelman, J.W. (1974): Clinical chemistry principles and techniques. 2nd Edn. Lange Medical Publication.

Hombal, P.R.; Gudadappanavar, A.M. and Javali, S.B. (2017): Association between serum linezolid concentration and haematological toxic effects: a longitudinal study. International Surgery Journal, 4(11): 37463752.

Khanalizadeh, M. and Najafian, M. (2014): Studying the effect of vitamin $\mathrm{E}$ and selenium on liver enzymes in chemotherapy rat with cyclophosphamide. Biosciences biotechnology Research Asia, 11(2): 1031-1035.

Kind, P. and King, E. (1954): Calorimetric determination of alkaline phosphatase activity. J. Clin. Path, 7: 322.

Lau, B.H. (2001): Suppression of LDL oxidation by garlic. The Journal of nutrition, 131: 985S988S.

Mellon, F.; Self, R. and Startin, J.R. (2000): Mass spectrometry of natural substances in foods. The Royal Society of Chemistry, Cambridge, UK, p. 299.

Mohammed, N.M.; Abd, A.H. and Qasim, B.J. (2014): The nephroprotective effects of vardenafil against amikacin induced nephrotoxicity in rabbits. International Journal of Advanced Research, 2(11): 747-755.

Nakagawat, S.; Kasug, S. and Matsuura, H. (1989): Prevention of liver damage by aged garlic extract and its components in mice. Phytotherapy Research, 3: 50 -53.

Nasr, A.Y. (2014): Protective effect of aged garlic extract against the oxidative stress induced by cisplatin on blood cells parameters and hepatic antioxidant enzymes in rats. Toxicology Reports, 1: 682-691.
Newman, D.J. and Price, C.P. (2005): Renal function and nitrogen metabolites. In: Burtis CA, Ashwood ER, eds. Tietz textbook of clinical chemistry. Philadelphia: WB Saunders 1999: 1204-1270.

Nishikimi, M.; Rao, N.A. and Yagi, K. (1972): The occurance of superoxide anion radical in the reaction of reduced phenazine methosulphate and molecular oxygen. Biochemical Biophysical Research Communications, 46: 849-854.

Nukui, Y.; Hatakeyama, S.; Okamoto, K.; Yamamoto, T.; Hisaka, A.; Suzuki, H.; Yata, N.; Yotsuyanagi, H. and Moriya, K. (2013): High plasma linezolid concentration and impaired renal function affect development of linezolidinduced thrombocytopenia. Journal of Antimicrobial Chemotherapy, 68: 2128-2133.

Ohkawa, H.; Ohishi, N. Yagi, K. (1979): Assay for lipid peroxidation in animal tissues by thiobarbituric acid reaction. Annals of Biochemistry, 95: 351-358.

Perrone, R.D.; Madias, N.E. and Levey, A.S. (1992): Serum creatinine as an index of renal function: new insights into old concepts. Clinical chemistry, 38: 1933-1953.

Reitman, S. and Frankel, S. (1957): A colorimetric method for the determination of serum glutamic oxalacetic and glutamic pyruvic transaminases. Am. J. Clin. Pathol, 28: 56-63.

Salem, N.A. and Salem, E.A. (2016): Protective Antioxidant Efficiency of Garlic against LeadInduced Renal and Testicular Toxicity in Adult Male Rats. J. Heavy Met Toxicity Dis, $1: 3$.

Schäfer, G. and Kaschula, C.H. (2014): The immunomodulation and anti-inflammatory effects of garlic organosulfur compounds in cancer chemoprevention. Anti-Cancer Agents in Medicinal Chemistry, 14: 233-240.

Shaarawy, S.M.; Tohamy, A.A.; Elgendy, S.M.; Elmageed, Z.Y.; Bahnasy, A.; Mohamed, M.S.; Kandi, E. and Matrougui, K. (2009): Protective effects of garlic and silymarin on NDEA-induced rats hepatotoxicity. International Journal of Biological Science, 11,5(6): 549-557.

Shirzad, H.; Taji, F. and Rafieian-Kopaei, M. (2011): Correlation between antioxidant activity of garlic extracts and WEHI-164 fibrosarcoma tumor growth in BALB/c mice. Journal of medicinal food, 14(9): 969-974

Sokol, R.J. (1996): Antioxidant defenses in metal induced liver damaged. Seminars in Liver Disease, 16 (1): 39-46.

Takahashi, Y.; Takesue, Y.; Nakajima, K.; Ichiki, K.; Tsuchida, T.; Tatsumi, S.; Ishihara, M.; Ikeuchi, H. and Uchino, M. (2011): Risk factors associated with the development of thrombocytopenia in patients who received 
linezolid therapy. Journal of Infection and Chemotherapy, 17 (3): 382-387.

Tamhane, A. and Dunlop, D. (2000): Statistics and data analysis from Elementry to intermediate prentice Hall. Upper Saddle River, USA.

Tripati, K.D. (2014): Essential of Medical Pharmacology, $7^{\text {th }}$ ed. New Delhi, India: Jaypee Brothers Medical Publishers, 752-764.

Tsai, W.T.; Chen, H.W.; Sheen, L.Y. and Lii, C.K. (2012): Garlic: Health benefits and actions. BioMedicine, 2: 17-29.

Uma, D.P.; Murugan, S.; Suja, S.; Selvi, S.; Chinnaswamy, P. and Vijayanand, E. (2007): Antibacterial, In vitro Lipid per Oxidation and Phytochemical Observation on Achyranthes Bidentata Blume. Pakistan Journal of Nutrition, 6 (5): 447-451.

Vinh, D.C. and Rubinstein, E. (2009): Linezolid: a review of safety and tolerability. Journal of Infection; 59(S1): 59-74.

Vivekanandan, L.; Sheik, H.; Singaravel, S. and Thangavel, S. (2018): Linezolid Therapy and its Associated Complications in MethicillinResistant Staphylococcus aureus (MRSA) Infected Diabetic Rats. International Journal of Pharmaceutical Sciences Review and Research, 52(1): 68-74.

Wang, X. and Quinn, P. (1999): Vitamin E and its Function in Membranes. Progress in lipid research, 38: 309-336.

Zaidi, S.K.; Ansari, S.A.; Tabrez, S.; Ashraf, G.M.; Shakil, S.; Jafri, M.A.; Naseer, M.I.; Abdulaal, W.H.; Banu, N. and Al-Qahtani, M. (2019): Hepato-protective effect of Allium sativum against immobilization stress in rats. Pakistan Journal of Pharmaceutical Sciences, 32(2): 521-528.

Zwart, L.L.; Meerman, J.H.; Commandeur, J.N. and Vermeulen, N.P.E. (1999): Biomarkers of free radical damage: applications in experimental animals and in humans, FreeRad Biol. Med., 26: $202-226$.

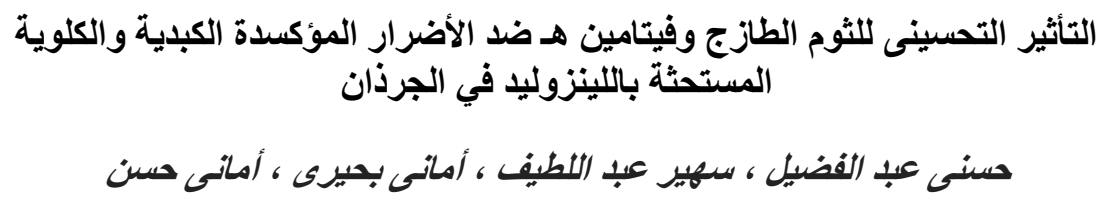

E-mail: amanybehairy25688@gmail.com Assiut University web-site: www.aun.edu.eg

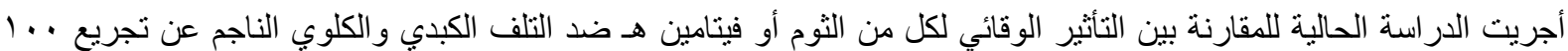

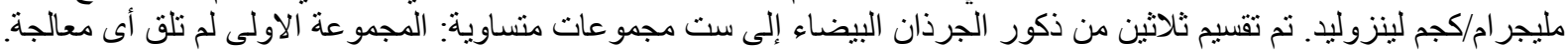

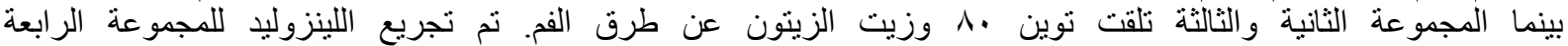

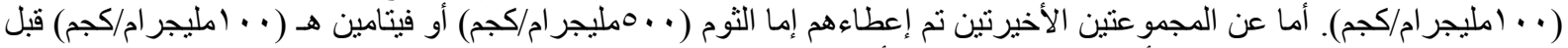

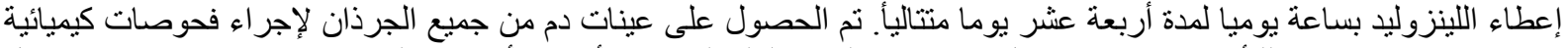

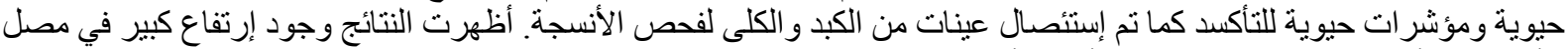

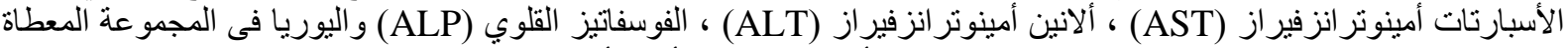

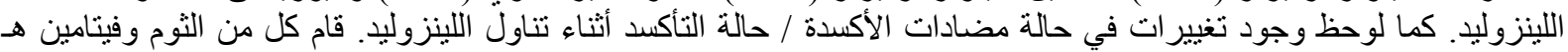

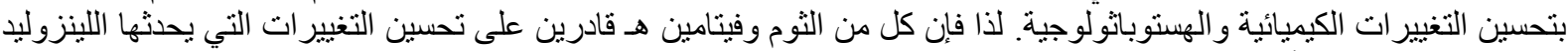
عبر آثار هم المضادة للأكسدة. 